\title{
Nanostructuring Materials for Spin Electronics
}

\author{
B. Doudin, J-Ph. Ansermet \\ Institut de Physique Expérimentale Ecole Polytechnqie Fédérale de Lausanne, PHB-Ecublens, \\ CH-1015 Lausanne
}

Fundamental studies on magnetic superlattices have sparked intense research activity worldwide. This is now leading to the development of devices based on the transport of electronic spins

\section{When magnetoresistance turned GIANT}

In the 1980 os there was an interest in the magnetic coupling between magnetic layers separated by non-magnetic layers.' This can be thought of as an extension of the concept of oscillations of the electron spin density around magnetic impurities in non-magnetic metals. Multilayers were indeed the site of similar oscillations and the layers experience a magnetic coupling mediated by the electrons of the nonmagnetic spacers. ${ }^{2,3}$ Furthermore, these multilayers were found to have a negative magnetoresistance (Fig. 1). ${ }^{4}$ As this novel effect was about an order of magnitude larger than the magnetoresistance of bulk ferromagnets, it was perceived as "giant". Relative changes over $100 \%$ of the resistance with magnetic field have been obtained. ${ }^{5}$ In bulk ferromagnets, a magnetoresistance results from the dependence of the resistivity on the orientiation of the current relative to the magnetization, the so-called anisotropic magnetoresistance (AMR). ${ }^{6}$ Although this AMR effect only contributes about $2 \%$, as in permalloy, it has been enormously useful. It has found applications in reading heads of magnetic recording devices, ${ }^{7}$ and in magnetic proximity or motion sensors.

From a talk presented at EPS-10, Trends in Physics, Symposium on the physics and chemistry of new materials (Prof. Sawatzky, chairman).

Dr. Bernard Doudin has established a laboratory for the production and the study of magnetic nanostructures produced by electrodeposition. He obtained a PhD in crystallography at the University of Lausanne.

J-Ph Ansermet is professor of experimental physics at EPFL. Formerly, he was at the Materials Research Center of Ciba-Geigy S.A. His PhD and post-doctoral work was carried out at the University of Illinois at Urbana-Champaign, where he developed NMR techniques for the study of surface phenomena.
Why magnetic layers separated by non-magnetic metals ought to exhibit a negative magnetoresistance

In a ferromagnet it is reasonable to assume that spins which are polarized parallel or antiparallel to the magnetization have different resistivities. Therefore we write the resistivities of spins parallel or antiparallel to the magnetization as:

$$
\rho_{\downarrow}=\rho(1-\beta) \quad \rho_{\uparrow}=\rho(1+\beta)
$$

This spin dependence of the resistivity is expected in the so-called Mott picture, whereby s electrons carry the current and scatter into $\mathrm{d}$ bands, which are split in a ferromagnet, so that the density of empty states available for scattering events differs for spin up and spin down.

Consider a system of two ferromagnetic layers, one in a parallel configuration, the other in an antiparallel alignment, and the electrons do not flip their spins going from one layer to the other.

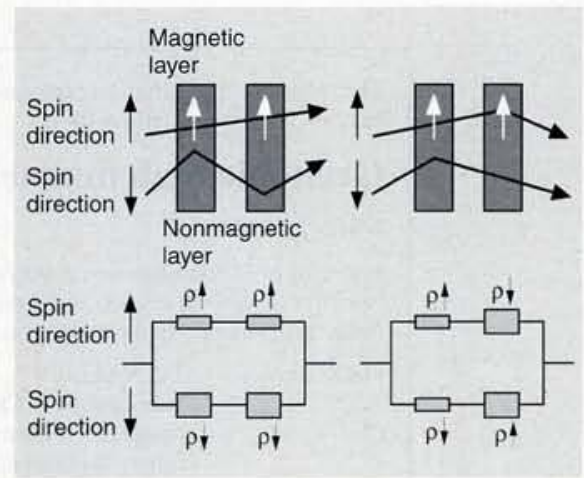

In the parallel configuration, one spin polarization experiences $\rho_{\uparrow}$ through both layers, the other spin polarization $\rho_{\downarrow}$ in both layers. The effective resistance is:

$$
\rho_{\downarrow \downarrow}=\rho_{\uparrow \uparrow}=\frac{2 \rho_{\uparrow} \rho_{\uparrow}}{\rho_{\uparrow}+\rho_{\uparrow}}=\rho\left(1-\beta^{2}\right)
$$

In the antiparallel configuration, each spin channel experiences $\rho_{\uparrow}$ and $\rho_{\downarrow}$ in series. The effective resistance is:

$$
\rho_{\uparrow \downarrow}=\frac{\rho_{\uparrow}+\rho_{\uparrow}}{2}=\rho
$$

The effective resistance is greater in the antiparallel configuration than in the parallel configuration, no matter what the sign of $\beta$ is. This model is directly inferred

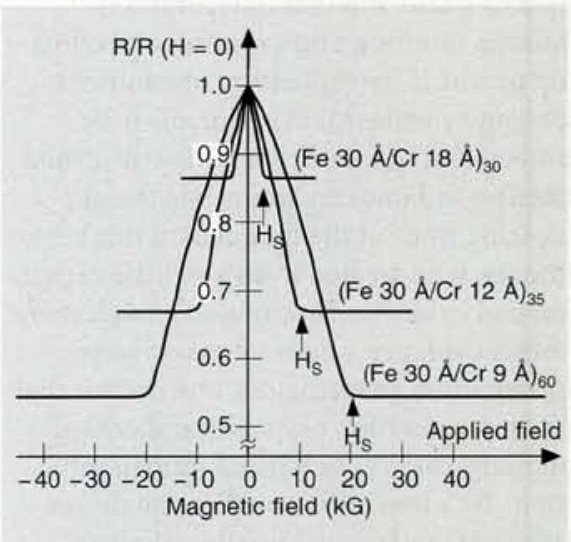

Fig.1. Magnetoresistance of a $\mathrm{Fe} / \mathrm{Cr}$ superlattice. This effect is now obtained at room temperature and fields of about a hundred Gauss. (from Baibich 1988)

from the two-current model, where the currents of both spin orientations are assumed separate. This is a reasonable model because a scattering event where the electron flips its spin while conserving its velocity is very rare.

Practically, in order to have a nonnegligible resistance value to observe, the measurements are usually performed in a geometry where the current flows parallel to the layers. If the layer thickness is larger than the electron mean free path, the simplest picture shown above fails and the giant magnetoresistance is severly diminished. Therefore, structuring on a nanometer scale is necessary.

\section{Engineering GMR materials}

Typically, superlattices or multilayers of a magnetic metal alternating with a non-magnetic metal have been used.

One way of getting the antiparallel alignment is to have the spacer layer just thin enough to produce a magnetic coupling mediated by the spacer (the socalled exchange coupling) which can be made antiferromagnetic. (Fig. 2a) 
Various GMR nanostructure (left) and their magnetoresistance behaviour (right - note that all the horizontal scales are different)

a Antiferrmagnetically coupled multilayer

b Spin - value structure

c Granular alloy

d Hybrid nanostructure including clusters and layers. See text for details.
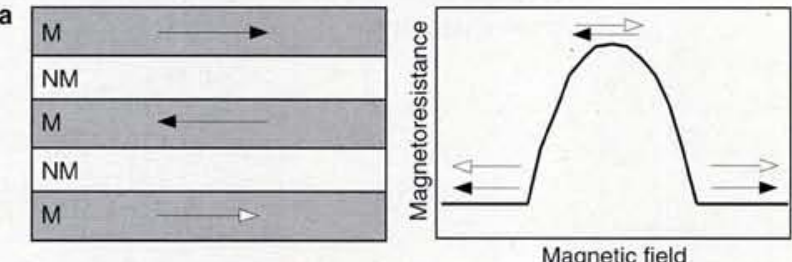

coupled multilayers

b
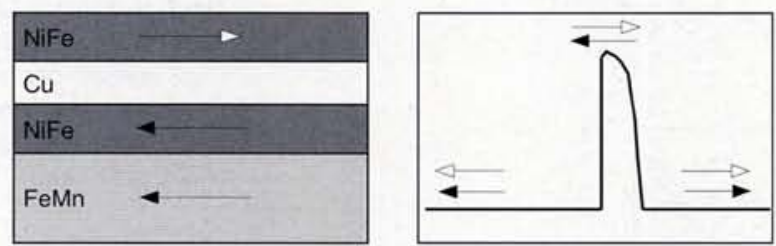

Spin valve structure

Granular material

Hybrid cluster/layer
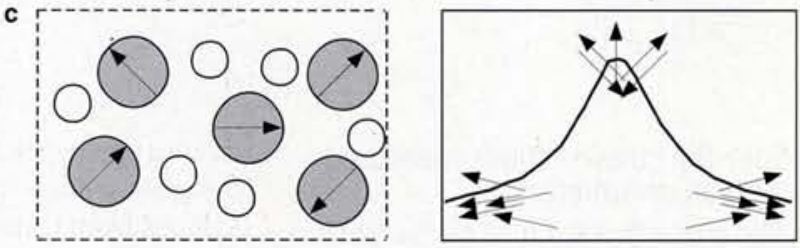

d
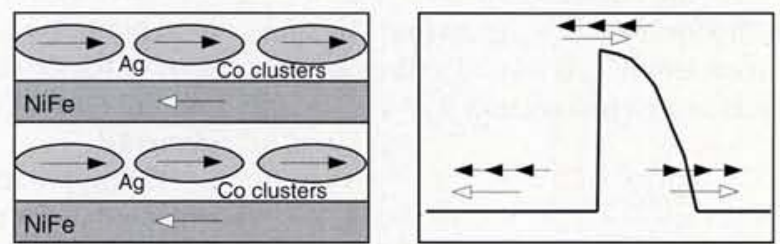

In the so-called spinvalve structure (Fig. 2b), one layer has its magnetization pinned, while the other is free to be reversed.

Granular materials with ferromagnetic ultrafine grains in a non-magnetic matrix (Fig. 2C) also show GMR. ${ }^{8,9}$

A remarkable result was obtained with a combination of Co clusters and permalloy layers spaced with silver (Fig. 2d). A record magnetoresistive sensitivity to the applied magnetic field of $6.5 \%$ per Gauss was achieved! ${ }^{\circ}$

\section{Perpendicular spin transport}

Whilst the two-current model used above predicts a "giant magnetoresistance", some of the actual issues require extensive research:

- How far can an electron travel before its spin flips?

- What is the relative importance of bulk and interface scattering in producing GMR ?
- What is spin dependent: the scattering potential itself, or the occupation of the states?

- Do the reflections of the electron wave functions at interfaces play a role?

Experiments in which the current was perpendicular to the interfaces have been particularly useful for shedding light on these issues. ${ }^{\text {" Perpendicular transport }}$ also has practical advantages over transport parallel to the interfaces: layers can be thicker when the current is perpendicular rather than parallel to the interfaces. This is relevant for practical applications, which must consider the ease of manufacturing and the reliability of the materials. The reason is the following. When the current is parallel to the layers the electrons experience two layers, provided their separation is of the order of the electron mean free path. When the current is perpendicular to the layers the spins undergo a diffusion process which extends over distances much longer that the electron mean free
Fig. 2. Nanostructures for GMR. From top to bottom: a) multilayers make use of an antiferromagnetic coupling among the layers; $\mathbf{b}$ ) spin valve bi-layer structures have one layer magnetically pinned; c) granular materials; d) hybrids. (after Physics World, Nov. 1994 p. 34)

path since the spin-flip events are rare. Indeed, electron spin resonance experiments in the 60 s and 70 s determined that electrons can travel a long way without flipping their spins.

\section{A tough challenge}

A small square cut from a thin film of a magnetic multilayer, with dimensions in the sub-millimeter range, has a resistance in the nano-ohm range! That measurement has been performed by a few..$^{12,13} \mathrm{Oth}$ ers have relied on advanced lithographic techniques to reduce the surface area and bring the resistance down to more accessible values. ${ }^{14}$ This is quite an extensive process. ${ }^{15}$ Moreover, when the columns are very thin, the current density is no longer homogeneous throughout the column. Furthermore, the field sensitivity is considerably reduced.

Instead of using advanced lithography to obtain thin columns, some groups produced multilayers in the form of wires by electrodeposition in pores of nanoporous membranes ${ }^{16,1718}$ (Fig. 3a). Others used grooved substrates and straight deposition (Fig. 3b), so that they measured GMR with current-at-an-angle (CAP) ${ }^{19}$. Or they deposited the layers obliquely to obtain a structure in which the current was mostly perpendicular to the layers ${ }^{20}$ (Fig. 3C).

\section{Nanowires of electrodeposited magnetic multilayers}

The electrodeposition of metals in the pores of polymeric membranes ${ }^{21}$ or in anodized aluminum ${ }^{22}$ is a well-established technique. The electrodeposition of thin film multilayers was demonstrated in the $80 \mathrm{~s}^{23}$ and magnetic multilayers with GMR were produced. ${ }^{24}$ It is only recently that it became established that one could grow multilayers in nanopores. The multilayered nanowires thus produced turned out to be very convenient for the study of GMR with current perpendicular to the plane.

By varying the layer thicknesses, it is possible to examine some of the basic issues of giant magnetoresistance (Fig. 4). Thanks to a model derived using the Boltzman equation ${ }^{25}$, the data can be interpreted in terms of: 


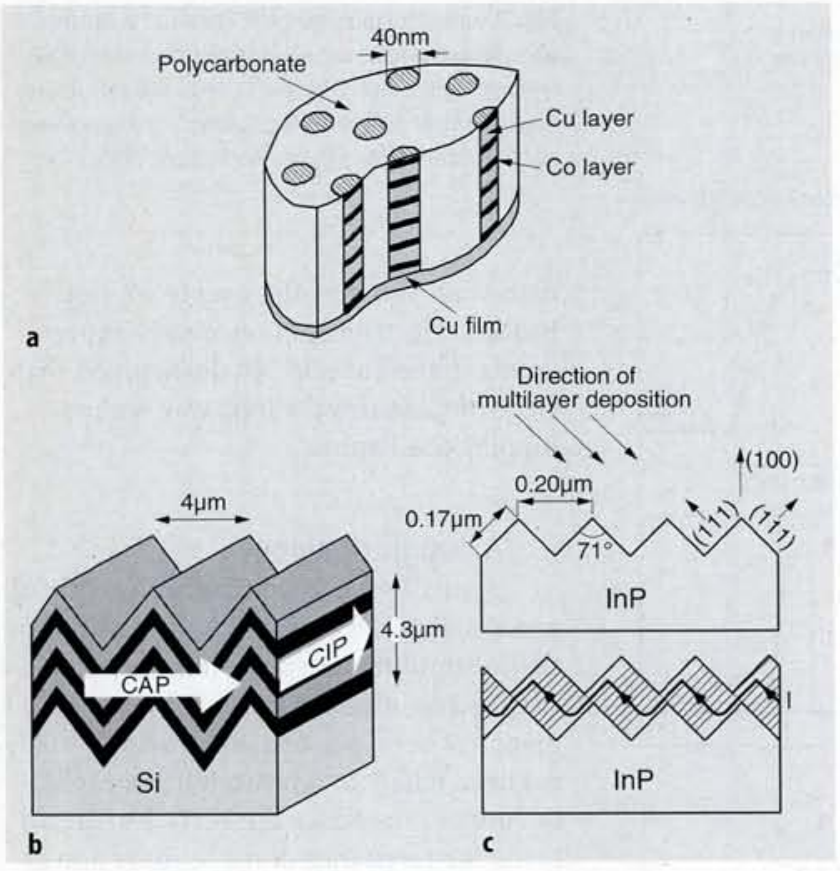

Fig. 3. Special ways of measuring spin transport perpendicular to the planes a) multilayered nanowires in nanoporous membranes, b) straight deposition and c) oblique deposition, on grooved substrates.
- bulk spin-dependent scattering, expressed as

$$
\rho_{\uparrow}=\rho(1-\beta) \quad \rho_{\downarrow}=\rho(1+\beta)
$$

- interface spin-dependent scattering, expressed as

$$
r_{\uparrow}=r 1(-\gamma) \quad r \downarrow=r(1+\gamma)
$$

- the spin diffusion length $l_{s f}$ which is a measure of the spatial extent of the diffusive process of spin-flip scattering, ensuring current continuity at the interfaces. This process is analogous to the build-up of excess charge at the interface between a normal and a superconducting metal. ${ }^{26}$

Our data yield the spin asymmetry coefficients $\beta$ and $\gamma$ and the spin diffusion length $l_{s f}$. Our results are compared with others in Table 1. There is remarkable consistency between the results of two groups using electrodeposited nanowires and one using vacuum deposition.

\section{Spin-flip cross-sections measured with an ohmmeter!}

The magnetoresistance measurements can yield the scattering cross sections for spin-flip for the following reason. The spin diffusion length $l_{s f}$ is related to the spinflip scattering cross section $\sigma_{s f}$ :

$$
l_{s f}=\sqrt{\frac{\lambda_{e}}{6 N \sigma_{s f}}}
$$

where $\mathrm{N}$ is the density of scattering centres and $\lambda_{e}$ the electron mean free path. $\lambda_{e}$ can be estimated from the resistivity of the $\mathrm{Cu}$ in these multilayers. $\mathrm{N}$ can be measured by energy dispersive $\mathrm{X}$-ray analysis. Hence from $l_{s f}$ we can deduce $\sigma_{s f}$.

The spin diffusion length of the electrodeposited $\mathrm{Co} / \mathrm{Cu}$ multilayers was ascribed to the presence of Co impurities in the $\mathrm{Cu}$ layers. The spin-flip scattering cross section of magnetic impurities in non-magnetic matrices was determined

\begin{tabular}{lllll}
$\beta$ & $\gamma$ & $\begin{array}{l}l_{s f}(\mathrm{Cu}) \\
(\mathrm{nm})\end{array}$ & $\begin{array}{l}l_{s f}(\mathrm{Co}) \\
(\mathrm{nm})\end{array}$ & ref \\
\hline 0.45 & 0.7 & 40 & $>10$ & 27 \\
0.4 & 0.8 & 140 & 40 & 28 \\
0.5 & 0.8 & varies with doping & 29
\end{tabular}

Table 1. Bulk and interface spin asymmetry parameters and spin diffusion lengths of $\mathrm{Co} / \mathrm{Cu}$ multilayers

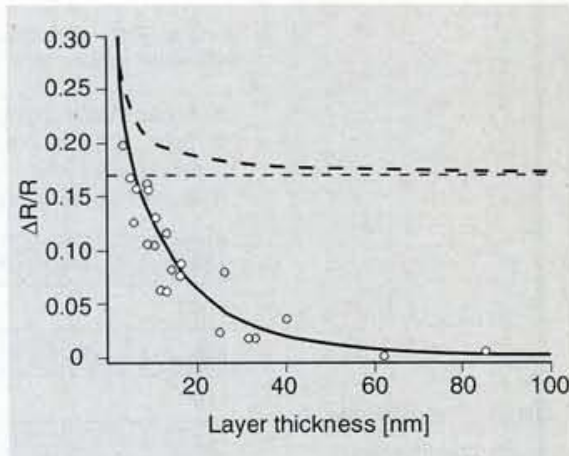

Fig. 4. Magnetoresistance $\Delta R / R_{\max }$ of $\mathrm{Co} / \mathrm{Cu}$ multilayers of equal $\mathrm{Co}$ and $\mathrm{Cu}$ thicknesses. Predictions for : (.......) bulk spin-dependent scattering only $\beta=0.4$ ); (- ) same, with interface scattering $\gamma=0.7$ ); $\left(\right.$ same, with spin diffusion effects $\left(l_{\mathrm{sf}}=40 \mathrm{~nm}\right)$. (data Doudin 1996)

decades ago by electron spin resonance ${ }^{30}$. The cross section for Co impurity in $\mathrm{Cu}$ deduced from transport measurements is consistent with these earlier results. Hence the appropriate nanostructuring has made possible the determination of electron spin-flip rates by a set of resistance measurements!

This approach offers new possibilities compared to the technique of transmission electron spin resonance, which is restricted by experimental conditions that can only be met in some cases. In particular, the spin-flip mean free path in ferromagnets such as Co had never been measured before. By keeping the $\mathrm{Cu}$ layers thin enough and varying the Co thickness of the multilayered nanowires, it has been possible to estimate it. ${ }^{28}$

\section{Solid state devices based on spin electronics}

There was a time of great excitement and productivity when vacuum tubes were replaced by solid state devices. Nowadays, as spin polarized vacuum experiments reach a high level of sophistication, solid state devices based on the spin of the electron are emerging from the fundamental research on spin transport in nanostructured materials. We evoke a few remarkable examples below.

Multilayers of giant magnetoresistance (with current flowing parallel to the interfaces) are expected soon to replace permalloy in the reading heads of magnetic recording devices. ${ }^{31}$ 
Magnetic bipolar transistors have been devised. ${ }^{32}$ Their concept arose out of a fundamental study of the phenomenon of spin injection from a ferromagnet into a non-magnetic metal. ${ }^{33}$ As in GMR, the transconductance of this transistor depends on the relative orientation of the magnetic layers.

Metal-gate field effect transistors ${ }^{34}$, where the gate is a magnetic multilayer with giant magnetoresistance, may be useful as field sensors because the collector current is an exponential function of the gate resistance. They are also of fundamental interest as the hot electrons jumping over the Schottky barriers probe the spin-dependent band structure above the Fermi level.

Magnetoresistive memories have been developed. ${ }^{31}$ Recent results may help improve such memories: tunnel junctions between two ferromagnetic layers have sharp, large resistance changes when the magnetization of the layers is switched from parallel to antiparallel. ${ }^{35}$

\section{Acknowlegments}

We are very grateful to A. Blondel and J. Meier, whose initial successes attracted the group Drs. G. Redmond, J.-E. Wegrowe, J. Tuaillon, S. Gilbert. Our work is supported by the Swiss NSF (grant no. 20-42034-92 to A.B., J. M.), the MINAST Swiss Priority Program (ELECPRO 7.08), the Brite-Euram program BR2-0546 (OFES support).

\section{References}

[1] "Materials Science and Engineering for the 1990 ", National Academy Press, Washington 1989, p. 95

[2] P. Grünberg, R. Schneider, Y. Pang, M.B. Brodsky, H. Sowers, Phys. Rev. Lett 57 (1986) 2442 [3] "Ultrathin Magnetic Structures I, II", Springer, Berlin 1994

[4] Baibich M:N., Broto J.M., Fert A., Ngyuen van Dau F., Petroff F., Etienne P., Creuzet G., Friedrich

A., Chjazelas J., Phys. Rev. Lett 61 (1988), 2472

[5] Levy, P.M., Solid State Physics Series, 47 (1994) 367

[6]T.R. McGuire, R.I. Potter, IEEE Trans. Magn. 11 (1975) 1018

[7] "Ultrahigh-Density Information Storage

Materials", MRS Bulletin 21 (9), September 1996

[8] J.X. Xiao, J.S. Jiang, C.L. Chien, Phys. Rev. Lett $68(5)(1992), 3749$

[9] A.E. Berkowitz, J.R. Mitchell, M.J. Carey, A.P. Young, S. Zhang, F.E. Spada, F.T. Parker, A. HJutten, G. Thomas, Phys. Rev. Lett 68 (25) (1992) 3745

[10] Holody-P., Steren-LB., Morel-R., Fert-A., Loloee-R., Schroeder-PA., Physical Review B-

Condensed Matter 50 1994, Iss 17, pp 12999-13002 [11] M.A.M. Gijs, G.E.W. Bauer, "Perpendicular Giant Magnetoresistance of Magnetic Multilayers", to be published in "Advances in Physics"

[12] W.P. Pratt Jr., S.F. Lee, J.M. Slaughter, R.

Loloee, P.A. Scroeder, J. Bass, Phys. Rev. Lett. 66 (1991) 3060

[13] P. Dauguet, P. Gandit, J. Chaussy, J. Appl. Phys. 79 (1996), 5823

[14] M.A.M. Gijs, J.B. Giesbers, S.K.J. Lenczowski, Phys. Rev. Lett. 70 (1993) 3343

[15] W. Vavra, S.F. Cheng, A. Fink, J.J. Krebs, G.A. Prinz, Appl. Phys. Lett. 66 (1995), 2579

[16] A. Blondel, J.P. Meier, B. Doudin, J-Ph

Ansermet, Appl. Phys. Lett. 65 (1994) 3019

[17] L. Piraux, J.M. George, J.F. Despres, C. Leroy,
E. Ferain, R. Legras; K. Ounadjela, A. Fert, Appl. Phys. Lett. 65 (1994) 2488

[18] K. Liu, K. Nagodawithana, P.C. Searson, C.L. Chien, Phys. Rev. 51 (1985) 7381

[19] T. Ono, T. Shinjo, J. Phys. Soc. Jpn. 64 (1995) 363

[2o] M.A.M. Gijs, M.T. Johnson, A. Reinders, P.E. Huisman, R.J.M. van de Veerdonk, S.K.J.

Lenczowski, R.M.J. van Gansewinkel, Appl. Phys. Lett. 66 (1995) 1839

[21] C. Martin, Science 266 (1994) 1961

[22] D. Aimawlawi, N. Coombs, M. Moskovits, J. Appl. Phys. 70 (1991) 4421

[23] C.A. Ross, Ann. Rev. Mater. Sci. 24 (1994) 159

[24] M. Alper, K. Attenborough, R. Hart, S.J. Lane,

D.S. Lashmore, C. Younres, W. Schwarzacher, Appl. Phys. Lett. 63 (15) (1993) 2144

[25] T. Valet, A. Fert, Phys. Rev. B45 (1993) 8689

[26] A.B. Pippard in "NonEquilibrium

Superconductivity, Phons and Kapitza Resistance", NATO ASI Series, B65 (1981)

[27] B. Doudin, A. Blondel, J-Ph Ansermet, J. Appl. Phys. 79 (1996) 6090

[28] L. Piraux. S. Dubois, C. Marchal, J.M. Beuken, L. Filipozzi, J.F. Despres, K. Ounadjela, A. Fert, JMMM 156 (1996) 317-320

[29] Yang, Q., Holody P., Lee SF., Henry LL.,

Loloee R., Schroeder PA., Pratt Jr. WP., Bass J., Phys. Rev. Lett. 72 (1994) 3274

[30] P. Monod, S. Schutz, J. Physique 43 (1982) 393401

[31] Physics Today special issue : Magneto-

electronics, April 1995

[32] M. Johnson, Materials Science and Engineering B31 (1995) 199

[33] M. Johnson, R.H. Silsbee, Phys, Rev. 35 (1987) 4959

[34] D.J. Monsma, J.c. Loder, Th. J. A. Poma, B.

Dieny, Phys. Rev. Lett. 74 (26) (1995)

[35] J.S. Moodera, L.R. Kinder, J. Nowak, P. LeClair,

R. Meservay, Appl. Phys. Lett, 69 (5) (1996) 708

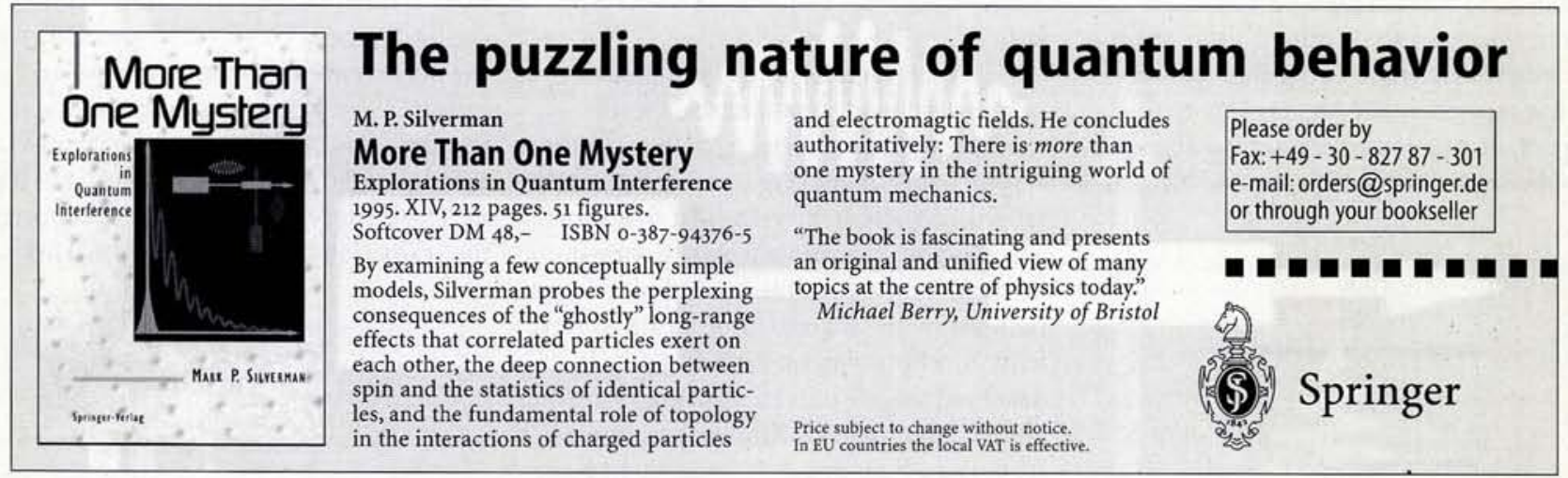

